\title{
Effect of Positional Release Therapy and Deep Transverse Friction Massage on Gluteus Medius Trigger Point - A Comparative Study
}

\author{
Doley $^{1}$, M, Warikoo ${ }^{2}$, D. \& Arunmozhi ${ }^{3}$, R. \\ ${ }^{1}$ Student Researcher, Department of Physiotherapy, Dolphin PG Institute of Biomedical \& Natural Sciences, \\ Dehradun. \\ ${ }^{\mathbf{2}}$ Assistant Professor, Department of Physiotherapy, Dolphin PG Institute of Biomedical \& Natural Sciences, \\ Dehradun. Email: deeptee.pt@gmail.com \\ ${ }^{\mathbf{3}}$ Associate Professor, Department of Physiotherapy, SBS PGI of Biomedical \& Research, Dehradun. Email: \\ rmozhi@gmail.com
}

\section{Abstract}

Study Objectives: To compare the effectiveness of Positional Release Therapy \& Deep Transverse Friction Massage On Gluteus Medius Trigger Point. Methods: 30 subjects were randomly recruited from various hospitals and community center in Dehradun and Guwahati based on the inclusion and exclusion criteria. The subjects were divided into two Groups (PRT (Group A) \& DTFM (Group B). Outcome Measure: Pressure pain threshold. Results: Both Groups A and B shown significant improvement in pressure pain threshold when comparison is made within the group. However Group B shown significant improvement in pressure pain threshold $(\mathrm{p}=0.001)$ post intervention between the group. Conclusion: Deep transverse friction massage is better choice of treatment in improving pain threshold in subjects with gluteus medius trigger point.

Key Words: Myofascial Trigger Point, Myofascial Pain Syndrome, Strain Counter Strain, Pressure Pain Threshold.

\section{Introduction}

Myofascial trigger points are extremely common and become a painful part of nearly everyone's life at one time or other. Myofascial trigger point is described as a hyperirritable spot in skeletal muscle that is associated with a hypersensitive palpable nodule in a taut band. Myofascial trigger points are the hallmark characteristics of Myofascial pain syndrome and feature motor, sensory, and autonomic components. Motor aspects of active and latent trigger points may include disturbed motor function, muscle weakness as a result of motor inhibition, muscle stiffness, and restricted range of motion. Sensory aspects may include local tenderness, referral of pain to a distant site (Dommerholt et al., 2006; Travell, 1999).
The formation of a myofascial trigger point may result from a variety of factors such as a severe trauma, overuse, overstress, psychological stress and joint dysfunction. Recent studies have hypothesized that the pathophysiology of Myofascial pain syndrome and the formation of Myofascial trigger point result from injured or overloaded muscle fibres leading to involuntary shortening and loss of oxygen and nutrient supply with increased metabolic demand on local tissues (Dommerholt et al., 2006; Fernandez $C$ et al., 2005 and Malanga, 2010). Simons and Travell suggested that trigger points in the muscle quadratus lumborum and gluteus medius are frequently found in low back pain. Trigger points in the gluteus medius muscle refer pain and tenderness along the posterior 
crest of the ilium, to the gluteal (posterior and lateral aspect) and sacral regions which is commonly identified as low back pain or lumbago (Simons, 1983; Travell, 1999).

Positional release technique or strain counterstrain technique (PRT or SCS) is a passive intervention aimed at relieving musculoskeletal pain and related dysfunction (Atienza Meseguer et al., 2006; Chaitow, 2002; Ambrogio, 1997). The classical description of this technique was made by Jones in 1981 who recommended the adoption of a position of comfort of dysfunctional tissue exhibiting tender points (Atienza et al., 2006). Dardzinski et al (2006) found that the strain counterstrain technique was effective in reducing pain and improving function in patients with localized myofascial pain syndrome. Wong et al (1994) reported that strain counter strain reduced sensitivity to palpation and increased strength in subjects exhibiting tender points in the hip musculature. Deep transverse friction massage (DTFM) is a technique used by James Cyriax and Gillean Russell to affect musculoskeletal structures of ligament, tendon and muscle to provide therapeutic movement over a small area (Prentice, 2002). The technique is applied at right angles to the fibres comprising the tissue containing the lesion in a relaxed and shortened position (Boyling, 1994; Prentice, 2002). Hong et al (1993) hypothesized that deep massage can offer effective stretching and mobilization of taut bands also reducing pain and tenderness of myofascial trigger point (Fernandez et al, 2006). Both PRT and DTFM found separately to be effective on reducing tenderness and pain evoked by trigger points. Thus we set out to examine which amongst these techniques is most effective in treating gluteus medius trigger point. Therefore the purpose of the present study was to compare the effects of Positional Release Therapy and Deep Transverse Friction Massage on Gluteus Medius Trigger Point.

\section{Materials \& Methods}

Thirty subjects were included from various hospitals and community in Dehradun and Guwahati based on the inclusion and exclusion criteria and divided into 2 groups Group A $(n=15)$, and Group B $(n=15)$. Subjects presenting with at least one area trigger point at gluteus medius muscle between the ages of $20-40$ years were included. Before participating, the subjects read and signed informed consent form that was approved by the Institutional review board. Pre intervention measurements of pressure pain threshold using algometer were carried out for each patient. All the subjects received interventions in alternate days for 3 days. The PPT value was measured on $3^{\text {rd }}$ day and 5 th day prior to treatment and the final reading was taken on $7^{\text {th }}$ day. Subjects were excluded from the participation like any sensory disturbances in the gluteus medius region, Diagnosed disc prolapse, History of any trauma or surgery of the lower back or hip region, Diagnosed fibromyalgia syndrome, If the subjects is undergoing any myofascial pain therapy in the past one month before the study, and Sacroiliac joint dysfunction.

Procedure: Positional release technique subjects were asked to lie prone 
with the therapist standing on the same side of the trigger point. Once the trigger point was palpated on the gluteus medius the therapist extend and abducts the hip and supports the patient's leg on the therapist thigh until reported pain reduces by $70 \%$. The hip is positioned in marked external rotation for trigger points located posterior to the mid axillary line and in internal rotation for those located anterior to mid axillary line. The position of comfort was held for $90 \mathrm{sec}$. After the release the subject was put back slowly and passively to the neutral position (Ambrogio, 1997; Chaitow, 2002).The same procedure was repeated for 5 times.

\section{Deep Transverse Friction}

Massage subjects were asked to lie prone with pillow placed under the thigh of the involved side. The treatment area was cleaned and dried before applying the technique. The treatment was applied by the therapist standing at the side of the patient. Treatment was given by thumb after the involved trigger point was palpated on the gluteus medius with the muscle in relaxed and shortened position (hip extension and abduction). The thumb was used by the therapist to apply friction across the fibre of the muscle. Transverse friction was applied with as much pressure as the patient tolerated and the therapist's thumb and the patient's skin was moved together as one. The treatment was applied for 10 minutes (Boyling and Palastanga, 1994).

Data was analysed by using SPSS software (version-13). Paired t-test was applied to compare the PPT within the groups. Independent t-test was applied to compare the PPT between the groups. The $\mathrm{p}$ value was set at $(\leq 0.05)$.

\section{Results \& Discussion}

Table 1.1: Mean and SD of Age for the subjects of Group A and Group B

\begin{tabular}{ccccc}
\hline \multirow{2}{*}{ Demographic } & \multicolumn{2}{c}{ Group A } & \multicolumn{2}{c}{ Group B } \\
\cline { 2 - 5 } & Mean & SD & Mean & SD \\
\hline \multirow{2}{*}{ AGE } & 26.47 & 3.31 & 28.07 & 5.90
\end{tabular}

Table 1.2: Comparison of mean value for PPT at $1^{\text {st }}$, $3^{\text {rd }}, 5^{\text {th }}$ and $7^{\text {th }}$ day within Group A and Group B

\begin{tabular}{ccccc}
\hline & \multicolumn{2}{c}{ Group A } & \multicolumn{2}{c}{ Group B } \\
\cline { 2 - 5 } PPT & $\mathrm{t}$ & $\mathrm{p}$ & $\mathrm{t}$ & $\mathbf{p}$ \\
\cline { 2 - 5 } $\mathbf{1}^{\text {st }} \mathrm{v}$ & -9.260 & 0.000 & -16.84 & 0.00 \\
$3^{\text {rd }}$ & & & & \\
$1^{\text {st }} \mathrm{v}$ & -12.36 & 0.000 & -9.136 & 0.00 \\
$5^{\text {th }}$ & & & & \\
$1^{\text {st }} \mathrm{v}$ & -12.91 & 0.000 & -17.62 & 0.00 \\
$7^{\text {th }}$ & & & & \\
\hline
\end{tabular}

Table 1.3: Comparison of mean value for PPT at $1^{\text {st }}$, $3^{\text {rd }}, 5^{\text {th }}$ and $7^{\text {th }}$ day between Group A and Group B PPT GROUP A Vs GROUP B

\begin{tabular}{ccc} 
& $\mathrm{t}$ & $\mathrm{P}$ \\
\cline { 2 - 3 } $1^{\text {st }}$ day & -1.280 & 0.211 \\
$3^{\text {rd }}$ day & -2.813 & 0.009 \\
$5^{\text {th }}$ day & -2.981 & 0.006 \\
$7^{\text {th }}$ day & -3.890 & 0.001 \\
\hline
\end{tabular}

The study showed that there is improvement in the pressure pain threshold after the intervention in both the groups as shown in Table 1.2 and Fig.1.1. This improvement in pressure threshold was found to be statistically significant with $\mathrm{p}=.001$. When comparison is made between the group, Group B (Deep Transverse Friction Massage) showed more improvement in pressure pain threshold as compared to Group A. Table 1.3 shows the mean value of the data collected at $1^{\text {st }}$ (pre) day had been found statistically insignificant $(\mathrm{p}$-value $=.211)$ and the data of $3^{\text {rd }}, 5^{\text {th }}$ and $7^{\text {th }}$ day which had been found statistically significant ( $p$ value $=.009, .006, .001)$ when compared between the group. Fig.1.2 and fig.1.3 
shows the improvement in the pressure pain threshold after the intervention between the Group A and Group B, which clearly indicate that there is significant improvement in Group B.

Discussion: Myofascial trigger points are extremely common and become a painful part of nearly everyone's life at one time or other (Dommerholt et al., 2006). Trigger point in gluteus medius muscle cause referred pain that is commonly identified as low back pain or lumbago. Its three trigger point regions together refer pain and tenderness primarily along the posterior crest of the ilium, to the sacrum and to the posterior and lateral aspect of the buttock. Pain and tenderness may extend to the upper thigh also (Prentice, 2002; Travell, 1999). After comparison Group B (Deep transverse friction massage (DTFM)) showed significant effect on improving the pain threshold in subjects with gluteus medius trigger point. We compared transverse friction massage with Positional release technique as some therapist claims that transverse friction technique places considerable strain on their hand. However, DTFM was found to be significantly effective than PRT.

\section{Transverse friction massage} involves the application of friction and pressure at depth to the offending lesion which is considered to be the cause of pain or reduced function (Cyriax, 1978). Force is applied perpendicular to the fibres in an attempt to separate each fibres, mechanically, promotes local hyperemia, analgesia, and reduction of adherent scar tissue to ligament, tendon and muscle structure. Deep transverse frictions cause the stimulation of nociceptive endings connected to $\mathrm{A} \delta$ fibres and mechanoreceptors found in soft tissues which are connected to large diameter $A \beta$ fibres. These large diameter fibres have an effect on cells in the posterior horn of the cord tending to inhibit forward transmission of the small-diameter nociceptive information, i.e. the 'pain gate' is closed. Hence it is suggested that presynaptic inhibition at cord level will modulate peripheral pain and reduce its perception (Watson, 1986; Bowsher, 1988). There may also be inhibition of neurotransmission exerted from higher centers, as the arrival of nociceptive stimuli at certain central inhibitory nuclei in the CNS (Raphe nuclei and periaqueductal area of grey matter in the midbrain) causes release of chemicals from neurons at cord level which block the action of nociceptive neurotransmitters (encephalin, endorphin). Consequently, in terms of modulation of pain, transverse frictions can be justified on both counts as they will cause presynaptic inhibition at the cord level and inhibit pain by the central production of encephalins (Boyling and Palastanga, 1994). Massage increases the blood circulation in the soft tissue, thus enhances the excretion of lactate or inflammatory substances and facilitate secretion of endogenous opiates (Yoon et al., 2012). This mechanism explains the reason for the results in our study where the pain threshold improved significantly in the DTFM group.

Macgregor et al (2012) suggested that massage resets sarcomere lengths. Deep cross-friction massage facilitates the proliferation of fibroblasts and results in the facilitation of soft tissue healing and realignment as pressure applied to the muscle is increased (Yoon et al., 2012). It 
is also reported that deep cross-friction massage was effective on subacute nonspecific low back pain (Farasyn \& Meeusen, 2007). Hong and colleagues (2006) reported that the best results in decreasing pain from myofascial trigger point were obtained with a deep pressure soft tissue massage. These results support our study where the pain threshold improved significantly in DTFM group. However the present study showed significant effect in Group A (positional release therapy) on pain threshold in within group. In support of present study Positional release technique is thought to achieve its benefits by means of an automatic resetting of muscle spindles which would help to dictate the length and tone into the affected tissues (Atienza Meseguer et al., 2006). Simons proposed that local pressure may equalize the length of the sarcomeres in the involved Trp (trigger point) and consequently decrease pain. Strain-counterstrain technique is usually applied with the targeted muscle in a shortened position, and used to treat tender points (Fernandez et al., 2006). It might be that pain relief from straincounterstrain technique may result from the stimulation of A $\delta$ fibres (Atienza Meseguer et al., 2006). These reasons supports the results of our study where the pain threshold improved in the PRT group. Wong and Schauer-Alvarez found in their study that strain-counterstrain reduced sensitivity to palpation in subjects exhibiting tender points in hip musculature (Atienza Meseguer et al., 2006). Dardzinski et al found that the strain counterstrain technique was effective in reducing pain and improving function in patients with localized myofascial pain syndrome (Dardzinski et al., 2006). Results of the present study demonstrates that group B showed statistically significant improvement in pressure pain threshold when compared to group A. Reduction in myofascial trigger point tenderness after the application of PRT could be due to the manual contact component of the treatment and also due to change in trigger point sensitivity rather than any unintentional release of pressure as suggested by Lewis $\mathrm{C}$ and Fryer and Hodgson in their study. On the other hand transverse friction massage not only results in the resets of sarcomere lengthening but it also helps in the proliferation of the fibroblast which thereby not only improves the soft tissue healing but also realign the muscle fibres by offering the effective stretching and mobilization to the taut bands. This mechanism could be the reason for better result yielded in the DTFM group

Limitations of the study are small sample size and no blinding was done in the study. Future study can be done with broader dimension, long term follow up and with the use of other outcome measure (range of motion and ODI scale).

Conclusion: The present study demonstrates that both the technique is effective in improving the pain threshold in subjects with gluteus medius trigger point. Our study highlights that deep transverse friction massage is better choice of treatment in improving pain threshold in subjects with gluteus medius trigger point. Acknowledgement: I am thankful to the Chairman, HOD and all the faculty members of the Dolphin PG Institute of Biomedical \& Natural Sciences, for their constant help and support during my 
research work. I am eternally grateful to the Chairman of Dolphin PG Institute of Biomedical \& Natural Sciences, Dehradun, for providing me the space and facilities during the research work.

\section{References}

Atienza Meseguer, A., Fernandez, C., NavarroPoza, J.L., Blanco, C.R., Gandia, J.J B. 2006. Immediate effect of strain counterstrain technique in local pain evoked by tender points. Clinical Chiropractic, 9: 112-118.

Boyling, J.D., Palastanga, N. 1994. Grieve's Modern Manual Therapy, The Vertebral Column. $2^{\text {nd }}$ edition. Churchill Livingstone, New York. 812-817.

Chaitow, L. 2002. Positional Release Therapy. $2^{\text {nd }}$ edition. Churchill Livingstone, Philadelphia, USA.

D Ambrogio, J., Roth, G. 1997 Positional Release. Assessment and Treatment of Musculoskeletal dysfunction. Mosby.167 \& 172.

Dardzinski, J A., Ostrov, B.E., Hamann, L S. 2006 Successful use of a strain counterstrain technique with physical therapy: Myofascial pain unresponsive to standard treatment. Journal of Clinical Rheumatology, 6(4): $169-74$

Dommerholt, J., Bron, C., Franssen, J. 2006. Myofascial Trigger point: An evidence informed review. The Journal of Manual and Manipulative Therapy, 14(4):230-221.

Farasyn, A., Meeusen, R. 2007. Effect of roptrotherapy on pressure pain thresholds in patients with subacute non-specific low back pain. Journal of Musculoskeletal Pain, 15: 41-53.

Fernandez, C., Blanco, C.A., Fernandez, J., Miangolarra, J. C. 2006. The immediate effect of ischemic compression technique and transverse friction massage on tenderness of active and latent myofascial trigger: a pilot study. Journal of Bodywork and MovementTtherapies, 10: 3-9.

Fernandez, C., Carnero, J. F. 2004. Transverse friction massage. A therapeutical alternative for the treatment of the myofascial pain syndrome. Fisioterapia, 26(3): 126-33.
Fernandez, C., Campo, M.S., Carnero, J.F. 2005. Manual therapies in myofascial trigger point treatment-a systemic review. Journal of Bodywork and Movement Therapies, 9: 2734.

Hong, C.Z., Chen, Y.C., Pon, C.H., Yu, J. 1993. Immediate effects of various physical medicine modalities on pain threshold of an active myofascial trigger point. Journal of Musculoskeletal Pain, 1(2): 37-53.

Malanga, G.A., Cruzcolon, E.J. 2010. Myofascial low back pain: A review. Physical Medicine Rehabiitationl Clinics of North America, 21: 711-724.

Njoo, K.H., Does, E.V. 1994 The occurrence and inter rater reliability of myofascial trigger points in the quadratus lumborum and gluteus medius : a prospective study in nonspecific low back pain patients and controls in general practice. Pain, 58: 317-323.

Prentice, W.E. 2002. Therapeutic Modalities for Physical Therapist. $2^{\text {nd }}$ edition. McGraw Hill Medical publishing division, New York. 430-431.

Simons, D.G., Travell, J.G. 1983. Myofascial origins of low back pain. Postgrad Med, 73(2): 99-105.

Stasinopoulos, D., Johnson, M I. 2004 Cyriax physiotherapy for tennis elbow/lateral epicondylitis. British Journal of Sports Medicine, 38: 675-677.

Travell, J., Simons, D. 1999. Myofascial pain and dysfunction. The trigger point manual. Upper half of body. $2^{\text {nd }}$ edition (Vol1). Lippincott Williams and Wilkins; 1-7, 7172, 19-21, 35, 116-121.

Travell, J., Simons, D. 1999. Myofascial pain and dysfunction. The trigger point manual. The lower extremities. $2^{\text {nd }}$ edition. ( Vol 2). Lippincott Williams and Wilkins; 150-165.

Yoon, Y.S., Yu, K.P., Lee, K.J., Kwak, S.H., Kim, J.Y. 2012. Development and application of a newly designed massage instrument for deep cross-friction massage in chronic nonspecific low back pain. Annals of Rehabilitation Medicine, 36: 55-65.

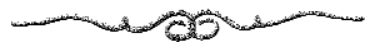

